\title{
GESTANTES EN CONTEXTOS FUNERARIOS ALTOMEDIEVALES NAVARROS
}

\author{
EXPECTANT MOTHERS IN EARLY MIDDLE AGES FUNERAL CONTEXTS FROM NAVARRE
}

\author{
Ma PAZ DE MIGUEL IBÁÑEZ \\ Universidad de Alicante \\ A Josefina Ibáñez Garrués, In Memoriam
}

\section{INTRODUCCIÓN}

En los últimos tiempos las excavaciones arqueológicas han sacado a la luz un gran número de necrópolis, en las que la realización de un minucioso trabajo ha permitido documentar la presencia de esqueletos de mujeres fallecidas durante la gestación.

El marco cronocultural de las Jornadas realizadas en Alicante, del que son fruto varios artículos de esta revista, centraba su interés en el tránsito entre el mundo tardoantiguo y los primeros momentos de la islamización. Por esta razón presentamos los datos obtenidos en tres necrópolis navarras, pertenecientes a este marco cronológico.

La primera de ellas, Gomacin (Puente la Reina), ha sido adscrita a la época tardoantigua, hispanogoda o visigoda, mostrando un ritual funerario claramente cristiano (Beguiristain, 2007, 203-204). La segunda, la maqbara de la Plaza del Castillo (Pamplona), fue utilizada durante el siglo VIII, momento del que se tienen referencias de la presencia de población musulmana en Pamplona (Unzu, 2004; Faro et alii, 2007; Faro, García-Barberena y Unzu, 2007; De Miguel, 2007). La tercera, la de la calle Herrerías (Tudela), tiene sus inicios en el siglo IX, prolongándose su uso hasta el siglo XI (Bienes, 2007a, 213; idem, 2007b, 257-258). Estas dos últimas presentan un rito funerario característico de la cultura islámica, con la presencia de los esqueletos en decúbito lateral derecho, mirando al este o sureste, en el interior de una fosa simple y careciendo, en general, de ajuar acompañante.

Son pocos los casos documentados de restos esqueléticos identificados como mujeres muertas durante el embarazo, aunque como veremos cada vez hay nuevos datos en las necrópolis que están siendo excavadas con minuciosidad, y en las que la conservación de los esqueletos permite evidenciar la presencia de restos fetales, muchas veces muy alterados por procesos tafonómicos. En épocas anteriores la investigación no daba relevancia a los esqueletos, quizás por falta de conocimiento respecto a la información en ellos contenida, o pudiera ser también ante la falta de personas especializadas que bien desde la Arqueología, la Antropología o la Paleopatología, ofrecieran una información adecuada a partir del estudio de estos materiales. Este hecho llevó a que no se prestara adecuada atención a los esqueletos, por lo que no se aplicó adecuadamente el tratamiento metodológico necesario a la hora de excavar y documentar tanto las alteraciones patológicas, como la existencia de individuos diferentes en la misma sepultura. Consideramos que siempre ha habido profesionales de la arqueología que han mostrado gran sensibilidad hacia este tema, primando una buena excavación y documentación a la premura con la que, en ocasiones, se les exige excavar un contexto funerario. Fruto de esos esfuerzos es el estudio que aquí presentamos.

La mortalidad materno-fetal se ha creído que debía ser importante en tiempos en los que los avances en obstetricia distaban mucho de los actuales. No obstante, el hallazgo de restos de mujeres gestantes en necrópolis puede ser considerado anecdótico. Es cierto que en ocasiones la aparición de esqueletos de gestantes al ser muy escasos numéricamente, condiciona que no se publiquen hasta tener finalizado el estudio general, por lo que estamos seguras de que los datos disponibles en la bibliografía no son el total de los documentados durante las excavaciones. Animamos a que sean publicados con el fin de conocer la incidencia real, y los nuevos datos que nos puedan aportar (edades maternas, posiciones fetales, edad gestacional, etc.), con el fin de aproximarnos al impacto real de la gestación respecto a la mortalidad materno-fetal.

Quisiéramos agradecer a M. Amor Beguiristain, Mercedes Unzu, José Antonio Faro y Juan José Bienes, la oportunidad de estudiar estos materiales, y su generosidad a la hora de ofrecernos toda la información disponible, parte de la cual ya había sido publicada en artículos precedentes. 


\section{CONTEXTOS HISTÓRICOS}

La necrópolis de Gomacin se localiza en el municipio navarro de Puente la Reina, siendo excavada por M. Amor Beguiristain en 1995. En ella se documentaron tres sepulturas, parcialmente alteradas por las labores agrícolas. Las sepulturas son cistas de lajas laterales conservando en una de ellas la tapadera de piedra. Se encontraban orientadas una E-O y dos N-S, con los esqueletos en decúbito supino. Se constata la reutilización de la sepultura 1, hecho frecuente en diferentes contextos funerarios altomedievales del entorno (Mezquíriz, 1965; Ramos, 2007; Faro y Unzu, 2007; Faro et alii, 2007). Los materiales asociados y las tipologías de las sepulturas, además de su localización geográfica han permitido asignar su pertenencia a época visigoda (Beguiristain et alii, 2001; Beguiristain, 2007).

Las sepulturas excavadas en la necrópolis fueron estudiadas por M. Amor Beguiristain, F. Etxeberria y L. Herrasti, habiendo publicado los resultados obtenidos (Beguiristain et alii, 2001). Destaca de esta necrópolis el caso de una mujer con signos claros de haber padecido lepra, siendo de interés el hecho de haber sido atendida por su entorno familiar de forma cuidadosa como indica su contexto funerario, sin haber sufrido un tratamiento diferenciado, a pesar de que las alteraciones producidas por la lepra fueron evidentes durante su vida (Etxeberria et alii, 1999; Beguiristain et alii, 2001, 264; Beguiristain, 2007).

La sepultura 1, construida con lajas y sin cubierta conservada, contenía restos de tres esqueletos, uno de ellos, hombre adulto, en conexión anatómica. El esqueleto del segundo individuo, perteneciente a una mujer adulta que se encontró parcialmente desarticulado, si bien aun conservaba en la zona de la pelvis restos de un individuo perinatal, llevando a inferir que se trataba del esqueleto de una mujer fallecida durante la gestación (Beguiristain et alii, 2001, 242; Beguiristain, 2007, 204).

La maqbara de la Plaza del Castillo ha supuesto un hito en el conocimiento de la ocupación islámica de la ciudad de Pamplona (Unzu, 2004; Faro et alii, 2007; Faro, García-Barberena y Unzu, 2007; De Miguel, 2007). Es bien conocido que en la investigación histórica el siglo VIII es un periodo del que apenas disponemos de fuentes escritas que nos permitan conocer de primera mano los acontecimientos ocurridos en el solar peninsular. La parquedad de las fuentes ha hecho que carezcamos de testimonios escritos directos que nos permitan conocer datos relevantes sobre los hechos acaecidos durante este periodo, particularmente en Pamplona. En este siglo el dominio islámico se impuso en todo nuestro territorio, incluido el norte peninsular. Pamplona pactó con el poder musulmán, con unas condiciones similares a las del pacto de Tudmir, de modo que el compromiso de cumplir con el pago de los tributos y de no oponerse al poder musulmán, favorecía la permanencia de las elites locales en el poder (Codera, 1903, 172; Cañada, 2002, 54). No obstante son varios los enfrentamientos que llevaron a la autoridad musulmana a tener que controlar diversas revueltas a lo largo del siglo VIII. De las pocas referencias conservadas disponemos de una de Ibn Idari al-Marrakusi (1999) en la que refiere que en tiempos de Uqba (734-741), ante las constantes revueltas en Pamplona, puebla la ciudad de musulmanes. Estos hechos justificarían el hallazgo de una necrópolis musulmana, con un perfil demográfico que nos muestra la presencia de una población estable en la ciudad, durante un periodo de tiempo impreciso, pero que posiblemente supere los 50 años, dada la cantidad de sepulturas exhumadas. Debemos señalar que la maqbara no está excavada en su totalidad, por lo que el número de sepulturas conocido es tan sólo una parte de la misma, y por el momento no podemos precisar la extensión de la zona que aun queda sin excavar.

Entre los restos esqueléticos exhumados se ha documentado la presencia de dos pertenecientes a mujeres fallecidas durante la gestación. La primera corresponde con la sepultura 119, con el esqueleto colocado en decúbito lateral derecho, comprobando que parte de los restos fetales se encontraban desplazados hacia el lado derecho, debido tanto al ritual islámico como a los procesos tafonómicos, circunstancia que condicionó la mezcla de los restos óseos fetales con los de las manos de la mujer, causando cierta confusión en su recogida (Fig. 1). En el interior de la pelvis se encontraron restos vertebrales, que confirmaron su identificación con una mujer embarazada.

El segundo caso, procedente de la sepultura 140, fue más complicado de identificar ya que el estado de conservación era más deficiente, y la confusión de los huesos de los dos individuos no permitió identificar la presencia del feto durante la excavación. La constatación de la presencia de restos de un perinatal junto con los de la mujer, nos hizo cribar el sedimento recogido y conservado procedente del interior de la pelvis. Gracias a ello se identificó la presencia de gérmenes dentales, un fragmento costal, y un cuerpo vertebral fetal (Fig. 2). Creemos que son argumentos suficientes para identificar el hallazgo como los restos esqueléticos pertenecientes a una mujer fallecida durante la gestación.

El tercer contexto estudiado es la Necrópolis de la calle Herrerías de Tudela, para la que se ha propuesto una cronología centrada en los siglos IX-XI, a partir de la presencia de algunos restos cerámicos (Bienes, 2006, 59). Durante la excavación realizada en 20052006, bajo la dirección de Juan José Bienes, se localizaron 236 enterramientos en decúbito lateral derecho mirando al S-SE, con una sola excepción en decúbito lateral izquierdo, mirando al SO. Hay que señalar la alta densidad de ocupación de la necrópolis, reflejada en la superposición de sepulturas, y la alteración de algunas de ellas al construir otras (Bienes, 2007, 254-255). En esta maqbara se han identificado varios niveles de enterramiento, indicadores de un uso prolongado del cementerio a lo largo del tiempo, o bien 


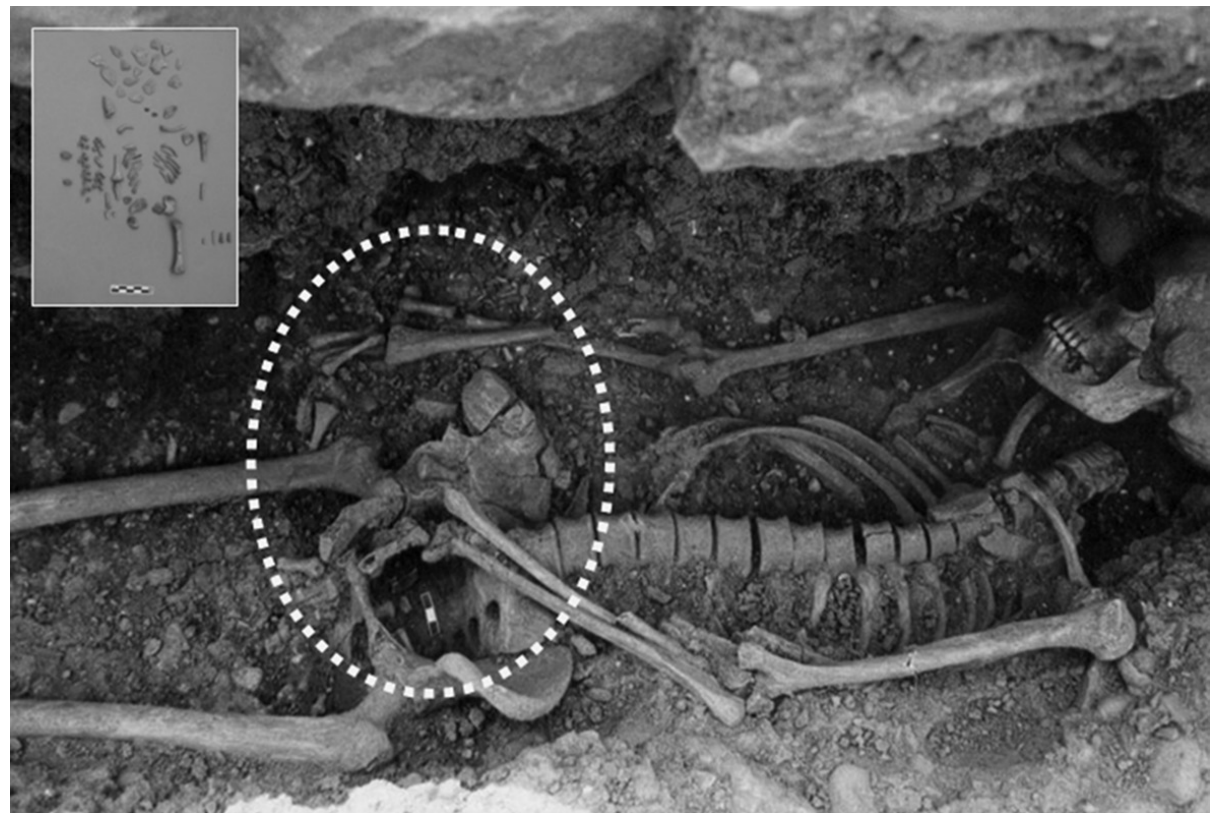

Figura 1: Plaza del Castillo, Sepultura 119 con los restos fetales conservados y su ubicación originaria.

fruto de un periodo de gran mortalidad, inferido indirectamente a partir de la presencia de algunas sepulturas múltiples sincrónicas, hecho claramente inusual en contextos islámicos (Bienes, 2006, 56; idem, 2007b, 257). No obstante, al menos una de ellas parece tener su origen en un momento de conflicto bélico, siendo seis los enterrados juntos, evidenciándose señales de violencia, causa más que probable de su muerte (Bienes, 2006, 58; idem, 2007, 257).

Por otra parte, fueron identificadas dos sepulturas en cuyo interior se hallaron los esqueletos de mujeres fallecidas durante el embarazo (Bienes, 2006, 53; idem, 2007, 256). En una de ellas la posición de los brazos delataba el avanzado estado de gestación en el que se encontraba, al intuirse un voluminoso abdomen que condicionó la clara separación de los brazos respecto de la pelvis (Fig. 3). La segunda presentó mayores dificultadas a la hora de su identificación y excavación debido a la gracilidad de los restos fetales.

Aunque se tienen noticias de otras necrópolis cristianas de esta época en Navarra (Azkarate, 2007, 178) no tenemos constancia de ningún esqueleto de gestantes en ninguna de ellas. Tenemos noticias de la aparición de embarazadas en otros contextos funerarios navarros, si bien sus cronologías se alejan un poco del

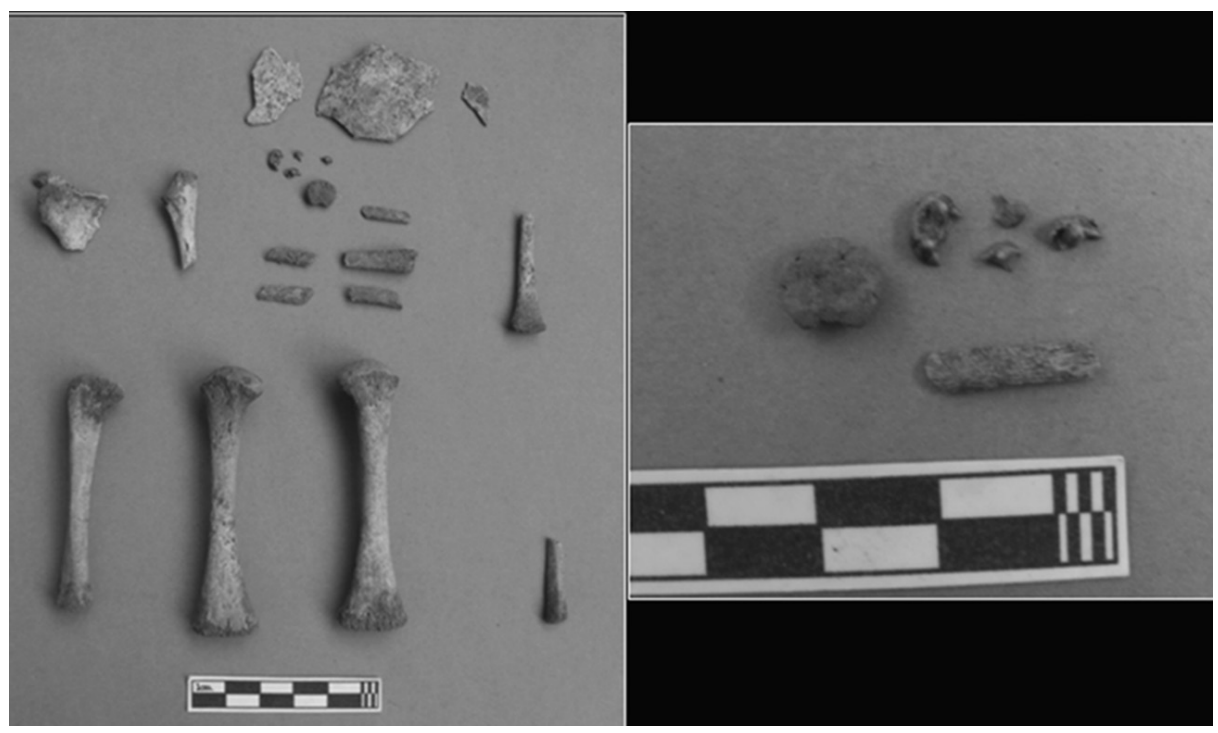

Figura 2: Restos fetales procedentes de la Sepultura 140 de la Plaza del Castillo. A la izquierda restos junto a la mujer, a la derecha restos junto al sedimento abdominal. 


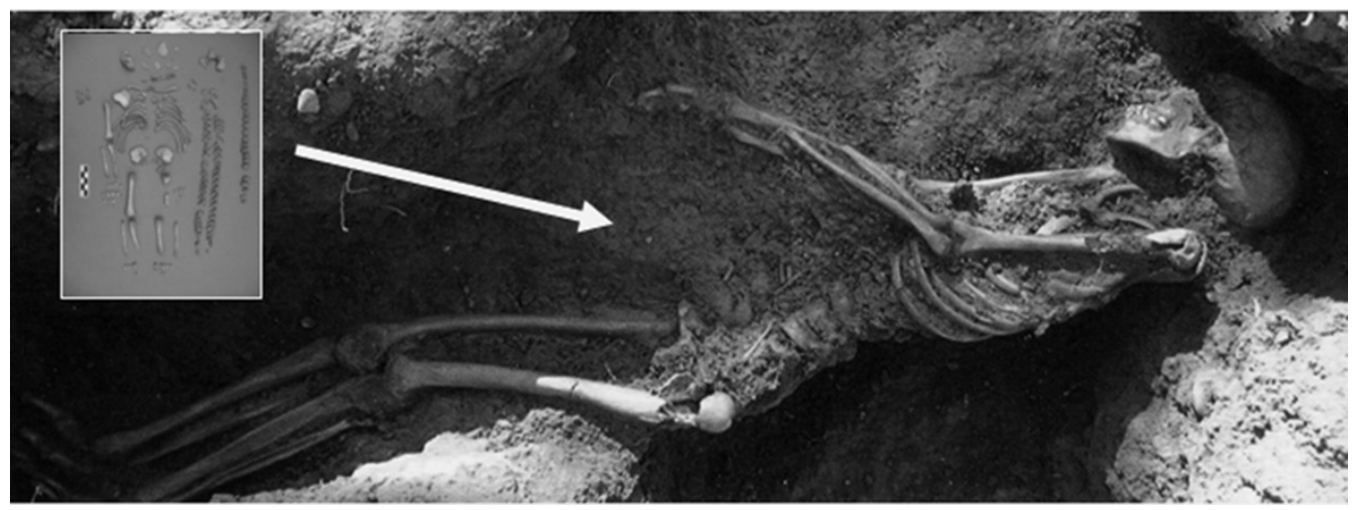

Figura 3: Calle Herrerías, Sepultura 184, junto a los restos fetales conservados (Fotografía de JJ Bienes, modificada).

marco cronológico en el que hemos centrado nuestro trabajo.

\section{METODOLOGÍA}

Tras la identificación, documentación y excavación de los esqueletos, se procedió a la limpieza de los huesos, en la medida de lo posible, así como a su restauración.

En el caso de los perinatales hemos utilizado las longitudes de los huesos largos, preferentemente del fémur, para hacer una aproximación a su edad en semanas de gestación. Hemos utilizado las tablas propuesta por Jeanty (Callen, 1997, 783), utilizando el percentil 50. Se ha contrastado con las propuestas de Fazekas y Kósa (1978). No se han determinado los sexos fetales, dadas las dificultades para aplicar las propuestas de diversos autores (Gil, 2000, 140-145), y las manifiestas posibilidades de error.

A partir de las fórmulas de Balthazard y Dervieux (1921) (en Reverte, 1999, 621), se han propuesto las tallas probables de los individuos. Somos conscientes de las limitaciones del método, si bien lo consideramos de interés para poder realizar comparaciones entre individuos.

Para los esqueletos de las mujeres, la determinación del sexo viene claramente determinada, en estos casos de forma irrefutable, por su evidente estado de gestación. No obstante, en todas ellas las características sexuales presentes tanto en la pelvis como en el cráneo, siempre que su estado de conservación así lo ha permitido, muestran signos claros de su correspondencia con una mujer (Buikstra y Ubelaker, 1994, 1521; Ferembach et alii, 1979).

La determinación de la edad en los esqueletos de las mujeres se ha realizado basándonos en las propuestas habituales: suturas craneales, desgastes dentales, signos degenerativos osteoarticulares, etc. (Buikstra y Ubelaker, 1994, 21-38; Brothwell, 1985, 108; Campillo, 2001, 55). En este caso no hemos considerado los datos ofrecidos por las sínfisis del pubis, ya que creemos que la existencia de embarazos altera las superficies de las sínfisis, lo que hace disminuir la validez del método.

No hemos pretendido hacer un estudio antropológico minucioso, si bien hemos obtenido algunos datos relacionados tanto con el Índice craneal, como con las posibles tallas en vida de las mujeres (Campillo y Subirá, 2004).

Otro aspecto relevante que hemos estudiado es la presencia de alteraciones patológicas en los esqueletos (Aufderheide y Rodríguez-Martín, 1998; Campillo, 2001; Isidro y Malgosa, 2003; Ortner, 2003). Es muy infrecuente encontrar alteraciones en los esqueletos fetales, ya que excepto en el caso de algunas malformaciones, y quizás de algunas infecciones, no ha habido margen temporal para que los huesos reflejen estar afectados por la enfermedad.

En el caso de los esqueletos de las madres nos hemos centrado en aquellas patologías dentales y óseas claramente identificables (caries, artrosis, etc.), ya que en principio debemos considerar que estamos ante mujeres jóvenes en las que no cabe esperar a priori graves alteraciones.

Se han documentado alteraciones entesopáticas relacionadas con actividades físicas forzadas (Capasso et alii, 1998). Igualmente, hemos identificado algunas variaciones de carácter epigenético (Buikstra y Ubelaker, 1994, 85-94).

\section{MATERIALES ESTUDIADOS}

\section{Gomacin (PUENTE LA ReINA)}

\section{Sepultura 1}

Adscripción crono-cultural: Necrópolis visigoda (s. VII). No disponemos de datación absoluta.

Descripción y conservación: Sepultura de lajas, en su interior se localizaron restos de tres individuos, un hombre en conexión anatómica, una mujer desplazada que conservaba parcialmente la suya, y restos fetales en el interior de su pelvis. 


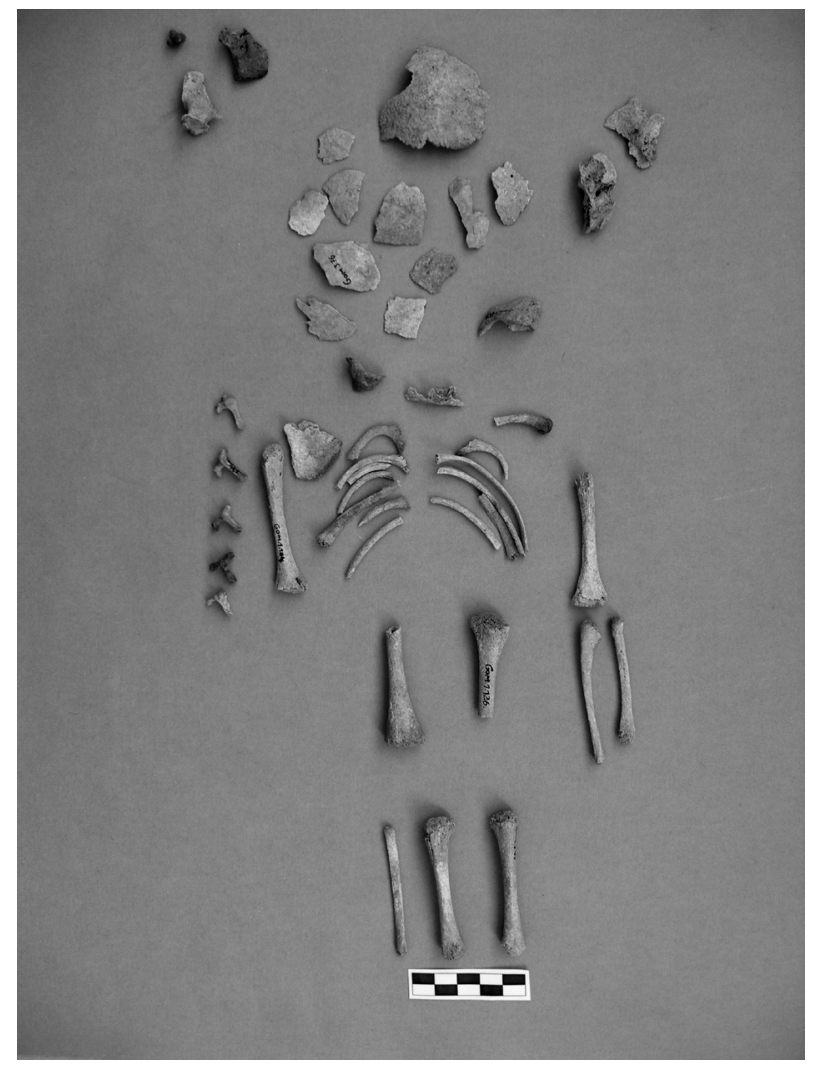

Figura 4: Fragmentos fetales de la Sepultura 1 de Gomacin.

Mujer: Parcialmente conservada, aunque el esqueleto está bastante bien representado.

Adulta joven (20-30 años) (Beguiristain et alii, 2001, 241).

Patología: Pérdida antemortem del diente 46. Caries en 47 y 36. Sarro en placa.

Talla: Manouvrier: 151-154 cm; Trotter y Gleser: $157 \mathrm{~cm}$.

Feto: \pm 40 semanas de gestación (Fig. 4).

Escasos fragmentos craneales. Fragmento mandibular, costillas, vértebras, clavículas y escápulas parcialmente conservadas; al igual que ambos húmeros, cúbito y radio izquierdos. Fragmentos de ambos fémures. Tibia y peroné casi completos.

Talla: $50^{\prime} 25 \mathrm{~cm}$.

\begin{tabular}{|c|c|c|}
\hline & \multicolumn{2}{|c|}{ Longitud máxima (mm) } \\
\hline & Derecho & Izquierdo \\
\hline Cúbito & -- & $61^{\prime} 5$ \\
\hline Radio & -- & $54^{\prime} 3$ \\
\hline Tibia & $65^{\prime} 1$ & 65 \\
\hline Peroné & $60^{\prime} 2$ & -- \\
\hline
\end{tabular}

\section{Plaza del Castillo (Pamplona)}

Adscripción crono-cultural: maqbara islámica. Disponemos de una datación absoluta sobre restos humanos (Beta: 218654. 660-770 dC. Cal. 2 sigmas). A partir del conocimiento histórico debemos centrar la cronología entre los años 714-715 y 770 dC.

\section{1.- Sepultura 119}

Descripción y conservación: Fosa simple con cubierta de lajas. Restos esqueléticos bien conservados. Algunas partes han sido reconstruidas.

Mujer; Adulta.

Patología: Sarro. Enfermedad periodontal. Osteítis en el alvéolo 46. Inicios de caries en 46. Osteoma en cóndilo mandibular derecho. Osteocondritis en cavidad glenoidea de la escápula derecha. Nódulo de Schmorl en la cara superior de L2. Ligera calcificación del ligamento amarillo en algunos arcos vertebrales torácicos.

Actividad: ligeras facetas de acuclillamiento en ambas tibias.

Variaciones epigenéticas: Rótulas ligeramente emarginatas.

Índice craneal: 74'01. Dolicocráneo.

Talla: Manouvrier: 145-146 cm; Trotter y Gleser: $147-$ $148 \mathrm{~cm}$.

Feto: \pm 38 semanas de gestación.

Parcialmente representado.

Talla: $48^{\prime} 8 \mathrm{~cm}$.

\begin{tabular}{|c|c|c|c|c|}
\hline & \multicolumn{2}{|c|}{$\begin{array}{c}\text { Longitud máxima } \\
(\mathbf{m m})\end{array}$} & $\begin{array}{c}\text { Anchura epífisis distal } \\
\text { (mm) }\end{array}$ \\
\hline & Derecho & Izquierdo & Derecho & Izquierdo \\
\hline Húmero & - & - & $16^{\prime} 4$ & - \\
\hline Fémur & - & $72^{\prime} 9$ & - & $18^{\prime} 6$ \\
\hline
\end{tabular}

\section{2.- Sepultura 140}

Descripción y conservación: Enterramiento en fosa simple. Buena conservación en general, algunos huesos han sido reconstruidos.

Mujer; Adulta.

Patología: Sarro. Enfermedad periodontal. Caries en 27 y 28 . Hipoplasia del esmalte en 33 y 34 . Artrosis en costillas. Ligera artrosis distal en cúbito izquierdo. Criba en cuellos femorales.

Actividad: Marcadas inserciones costo-claviculares. Ligera entesopatía en primeras falanges de las manos. Faceta de acuclillamiento en ambas tibias. Ligera entesopatía en las inserciones calcáneas del Tendón de Aquiles.

Variaciones epigenéticas: Sutura metópica persistente. Varios huesos wormianos en la sutura lambdoidea. Rótulas ligeramente emarginatas.

Índice craneal: 76’37. Mesocráneo.

Talla: Manouvrier: 154-155 cm; Trotter y Gleser: 155$156 \mathrm{~cm}$.

Feto: 40-41 semanas de gestación.

Parcialmente conservado. Algunos huesos largos completos.

Talla: $52^{\prime} 8 \mathrm{~cm}$. 


\begin{tabular}{|c|c|c|c|c|}
\hline & \multicolumn{2}{|c|}{$\begin{array}{c}\text { Longitud máxima } \\
(\mathbf{m m})\end{array}$} & \multicolumn{2}{c|}{$\begin{array}{c}\text { Anchura epífisis distal } \\
\text { (mm) }\end{array}$} \\
\hline & Derecho & Izquierdo & Derecho & Izquierdo \\
\hline Fémur & $78^{\prime} 4$ & $78^{\prime} 9$ & $20^{\prime} 4$ & $20^{\prime} 7$ \\
\hline Tibia & 69 & - & - & - \\
\hline
\end{tabular}

\section{CAlle de Herrerías (Tudela)}

Adscripción crono-cultural: maqbara islámica ss. IX-XI.

\section{1.- Sepultura 115}

Descripción y conservación: Enterramiento en fosa simple. Restos representativos. Conservación deficiente en general. Marcada destrucción tafonómica de las epífisis de los huesos largos.

Mujer; Adulta.

Patología: Osteoma en la parte inferior derecha del occipital (17'4 x 17’4 mm) (Fig. 5). Sarro. Pérdida dental antemortem de 27. Caries $(15,16,17,24$ y 36). Osteítis en el alvéolo 16 que afecta ligeramente al seno maxilar. Ligera impactación de 38 sobre 37. Escasa artrosis en cavidad glenoidea de la escápula izquierda. Artrosis costal. Artrosis ligera en caras superiores de T10, T11 y T12.

Actividad: Marcadas inserciones costoclaviculares en ambas clavículas. Entesopatía leve en las primeras falanges de las manos. Discreta faceta de acuclillamiento en la tibia derecha (izquierda no valorable).

Índice craneal: 73'22. Dolicocráneo.

Talla: Manouvrier: $149 \mathrm{~cm}$; Trotter y Gleser: $150 \mathrm{~cm}$.

Feto: 22-23 semanas de gestación.

Escasamente representado. La fragilidad de los restos justifica claramente su parcial conservación (Fig. 6).

Talla: $34 \mathrm{~cm}$.

Observaciones: Junto a los restos fetales se conserva una pequeña calcificación, posiblemente relaciona-

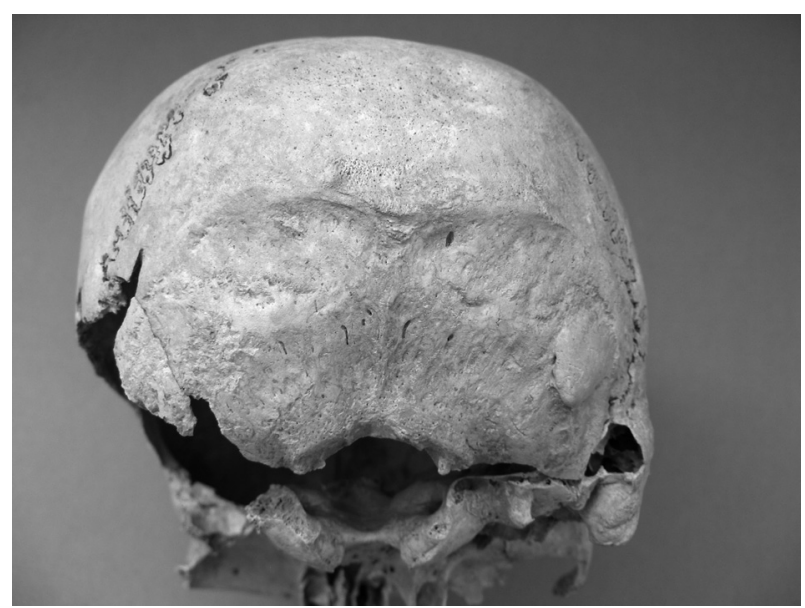

Figura 5: Osteoma occipital. Calle Herrerías 115.

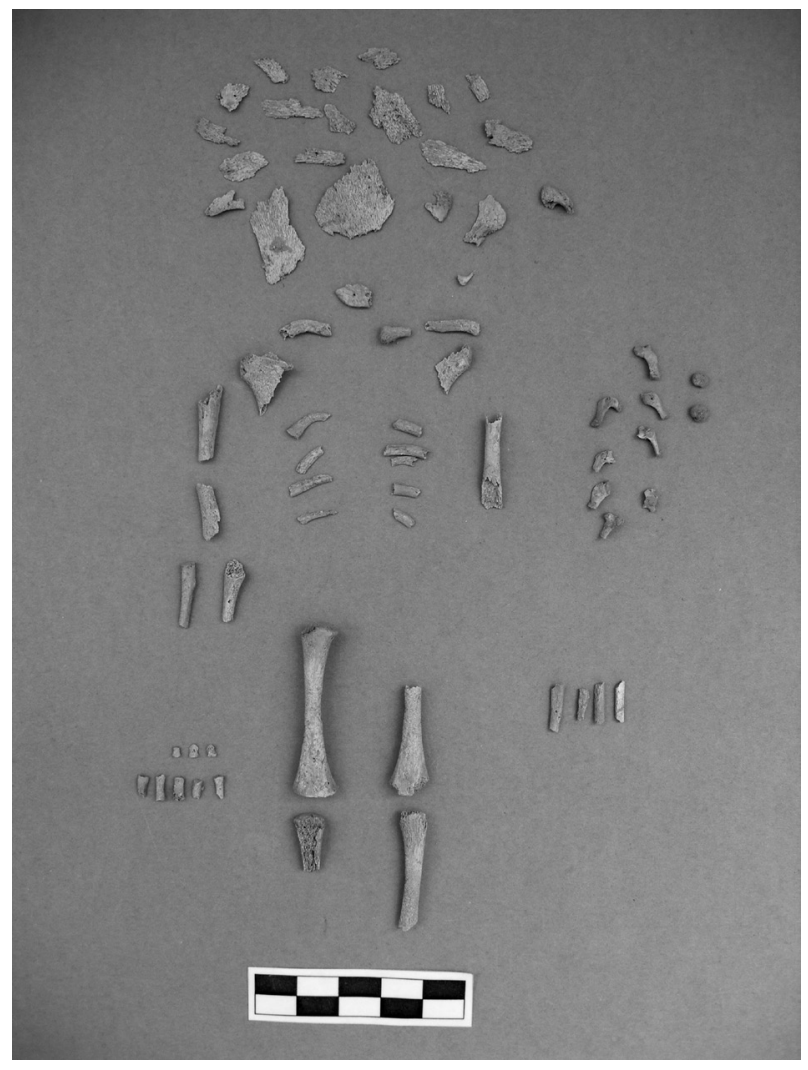

Figura 6: Feto inmadura de la sepultura 115 (Calle Herrerías).

da con la madre dado su tamaño (10’6 mm x 9'7 $\mathrm{mm} \times 5 \mathrm{~mm}$ ).

\begin{tabular}{|c|c|c|c|c|}
\hline & \multicolumn{2}{|c|}{$\begin{array}{c}\text { Longitud máxima } \\
(\mathbf{m m})\end{array}$} & $\begin{array}{c}\text { Anchura epífisis distal } \\
(\mathbf{m m})\end{array}$ \\
\hline & Derecho & Izquierdo & Derecho & Izquierdo \\
\hline Fémur & $40^{\prime} 1$ & - & $10^{\prime} 3$ & - \\
\hline
\end{tabular}

\section{2.- Sepultura 184}

Descripción y conservación: Enterramiento en fosa simple. Restos esqueléticos bien conservados, excepto el cráneo y las costillas.

Mujer; Adulta.

Patología: Sarro. Enfermedad periodontal. Caries en 26, con total destrucción de la corona y de parte de las raíces. Pérdidas antemortem de 16 y 25. Osteítis alveolar en la cara labial de los alvéolos mandibulares. Ligera artrosis en la cavidad glenoidea de la escápula derecha. Calcificación del ligamento amarillo entre T9 y T11, principalmente. Ligera epifisitis en el borde antero-superior de los cuerpos vertebrales de L3, L4 y L5. Ligero reborde osteofítico en la cara antero-inferior de L1. Nódulo de Schmorl en cara inferior de T11. Ligera artrosis en la articulación proximal de ambos cúbitos. Artrosis incipiente en la rótula izquierda.

Actividad: Marcadas inserciones del ligamento costoclavicular, bilateral. Faceta de acuclillamiento en 
ambas tibias. Ligera entesopatía en las inserciones calcáneas del Tendón de Aquiles. Surco lateral externo en ambos calcáneos.

Índice craneal: 67. Braquicráneo.

Talla: Manouvrier: 151-152 cm; Trotter y Gleser: 153 $\mathrm{cm}$.

Feto: \pm 38 semanas de gestación.

Buena conservación general.

Talla: 49'9 cm.

\begin{tabular}{|c|c|c|c|c|}
\hline & \multicolumn{2}{|c|}{$\begin{array}{c}\text { Longitud máxima } \\
\text { (mm) }\end{array}$} & $\begin{array}{c}\text { Anchura epífisis distal } \\
\text { (mm) }\end{array}$ \\
\hline & Derecho & Izquierdo & Derecho & Izquierdo \\
\hline Clavícula & 41 & $41^{\prime} 1$ & - & - \\
\hline Húmero & $\pm 61^{\prime} 3$ & - & $14^{\prime} 8$ & - \\
\hline Radio & $49^{\prime} 5$ & - & - & - \\
\hline Cúbito & $57^{\prime} 6$ & - & - & - \\
\hline Fémur & $\pm 70^{\prime} 3$ & - & $19^{\prime} 1$ & - \\
\hline Tibia & - & $63^{\prime} 8$ & - & - \\
\hline Peroné & 60 & \pm 59 & - & - \\
\hline
\end{tabular}

\section{VALORACIÓN DE LOS DATOS}

La investigación realizada en Navarra a partir de la excavación y estudio de diversas necrópolis pertenecientes a época altomedieval, tanto en contextos cristianos como musulmanes, nos ofrece información sobre la existencia de cinco mujeres fallecidas durante la gestación.

Son escasas las referencias, en los trabajos consultados, sobre la presencia de gestantes inhumadas (Fig. 7), si bien sabemos que son varias las que en la actualidad están en fase de estudio, por lo que esperamos su pronta publicación.

En diferentes trabajos se considera que la mortalidad materna relacionada con embarazos, partos y postpartos complicados debería dejar claro reflejo en los contextos funerarios (Mafart, 1994; Gómez Be1lard, 2001, 463; Rosemberg, 2005). No obstante, sólo a partir de la existencia de esqueletos de embarazadas pudiéramos inferir causas de muerte relacionadas con complicaciones obstétricas. Sólo en dos casos de cronologías más antiguas, como el del yacimiento de Coy (Lorca, Murcia) (Ayala, 1997; Malgosa et alii, 2004) con el feto en situación transversa lo que provocó un prolapso de brazo, y otro de la necrópolis tardorromana de Tarragona (Campillo et alii, 1998) donde se evidenció un parto de pies, se han documentado distocias de parto causantes, muy probablemente, de la muerte de la madre y el feto. Bien es cierto que la presencia en diversos yacimientos arqueológicos de fetos debe ser considerada como la evidencia de complicaciones relacionadas con el parto y el periodo perinatal inmediato, causantes del desarrollo de partos, muchas veces prematuros, en otros casos de gemelos, y en la mayoría de fetos a término, que pudieron desencadenar complicaciones maternas causantes de su muerte durante el postparto inmediato.

La valoración de la edad fetal nos permite sugerir las posibles complicaciones relacionadas con cada trimestre del embarazo. La presencia de fetos inmaduros, y por tanto incompatibles con la vida extrauterina, como ocurre en la sepultura 115 de la calle Herrerías de Tudela, nos hace pensar en complicaciones sistémicas de la mujer agravadas por el embarazo, infecciones, placenta previa, gestosis y desprendimiento prematuro de placenta, entre otros (Cararach, 1982).

En los casos de Gomacin, Plaza del Castillo (119 y 140) y calle Herrerías 184 las edades fetales corresponden con las de fetos a término, entre 38 y 40 semanas, sin evidencias documentadas de malposiciones fetales, por lo que consideramos como posibles causas obstétricas del óbito el desprendimiento prematuro de placenta, infecciones, gestosis, etc., no descartando las desproporciones pelvicocefálicas o la patología materna sistémica. El tamaño fetal no es indicador en ninguno de estos casos de una distocia de parto a excepción de la sepultura 119, con una talla materna media de entre 146-147 cm, que en ocasiones condiciona una desproporción pelvico-cefálica impidiendo el desarrollo normal del parto.

En el caso de la sepultura 140 de la Plaza del Castillo el tamaño del feto, a partir de los parámetros valorables como son la edad gestacional de 40-41 semanas y una talla de $52^{\prime} 8 \mathrm{~cm}$, pudiera indicarnos la presencia de un feto macrosoma, que presentara un mayor riesgo de desproporción pélvico-cefálica, y como consecuencia de la distocia, el fallecimiento de ambos.

A partir de los escasos datos disponibles tan sólo podemos presentar como posibles las causas obstétricas

\begin{tabular}{|c|c|c|c|c|}
\hline Yacimiento & $\begin{array}{l}\text { Adscripción } \\
\text { cronocultural }\end{array}$ & Edad materna & Edad fetal & Bibliografía \\
\hline $\begin{array}{l}\text { al-Fossar } \\
\text { Novelda (Alicante) }\end{array}$ & $\begin{array}{l}\text { Islámico } \\
\text { s. XIII }\end{array}$ & $\begin{array}{l}\text { Adulta joven } \\
\text { (20 años) }\end{array}$ & $31-32$ semanas & López Seguí et alii, 2005 \\
\hline $\begin{array}{l}\text { Baza } \\
\text { (Granada) }\end{array}$ & $\begin{array}{l}\text { Islámico } \\
\text { s. XI-XII }\end{array}$ & $\begin{array}{l}\text { Adulta } \\
\text { (21-25 años) }\end{array}$ & 29-31 semanas & Rascón et alii, 2007 \\
\hline $\begin{array}{l}\text { Puerta Elvira } \\
\text { (Granada) }\end{array}$ & $\begin{array}{l}\text { Islámico } \\
\text { s. XIII-XIV }\end{array}$ & Adulta & 36-38 semanas & De Miguel et alii, 2007 \\
\hline Sevilla & $\begin{array}{l}\text { Islámico } \\
\text { s. XII-XIII }\end{array}$ & Adulta & $36-40$ semanas & $\begin{array}{l}\text { López Flores y Magariño, } \\
2007\end{array}$ \\
\hline
\end{tabular}

Figura 7: Presencia de embarazadas en yacimientos altomedievales. 


\begin{tabular}{|c|c|c|c|c|c|c|c|}
\hline Yacimiento & \begin{tabular}{|c|}
$\begin{array}{c}\text { Adscripción } \\
\text { cronocultural }\end{array}$ \\
\end{tabular} & $\begin{array}{c}\text { Edad } \\
\text { Materna }\end{array}$ & $\begin{array}{l}\text { Talla } \\
(\mathrm{cm})\end{array}$ & $\begin{array}{c}\text { Índice } \\
\text { craneal }\end{array}$ & Patología & $\begin{array}{c}\text { Edad Fetal } \\
\text { (semanas) }\end{array}$ & $\begin{array}{l}\text { Talla } \\
(\mathrm{cm})\end{array}$ \\
\hline $\begin{array}{c}\text { Gomacin } \\
\text { Sepultura } 1\end{array}$ & $\begin{array}{c}\text { Visigodo } \\
\text { s. VII }\end{array}$ & Adulta & $\begin{array}{c}\text { M: } 153 \\
\text { T-G: } 157\end{array}$ & -- & $\begin{array}{l}\text { Pérdida antemortem } 46 \text {. Caries } 47 \text {, } \\
\text { 36. Sarro en placa }\end{array}$ & \pm 40 & $50 ’ 25$ \\
\hline $\begin{array}{c}\text { Plaza del } \\
\text { Castillo }\end{array}$ & $\begin{array}{l}\text { Musulmán } \\
\text { s. VIII }\end{array}$ & & & & & & \\
\hline $\begin{array}{l}\text { Sepultura } \\
119\end{array}$ & & Adulta & $\begin{array}{l}\text { M: } \\
\text { 145-146 } \\
\text { T-G: } \\
147-148\end{array}$ & 更 & $\begin{array}{l}\text { Sarro. Enfermedad periodontal. } \\
\text { Osteítis } 46 \text {. Inicios de caries en } 46 . \\
\text { Osteoma en cóndilo mandibular } \\
\text { derecho. Osteocondritis en } \\
\text { cavidad glenoidea. Nódulo de } \\
\text { Schmorl L2. Ligera calcificación } \\
\text { del ligamento amarillo }\end{array}$ & \pm 38 & $48^{\prime} 8$ \\
\hline $\begin{array}{l}\text { Sepultura } \\
140\end{array}$ & & Adulta & $\begin{array}{c}\text { M: } \\
\text { 154-155 } \\
\text { T-G: } \\
155-156\end{array}$ & $\begin{array}{c}76 \\
\text { Mesocráneo }\end{array}$ & $\begin{array}{l}\text { Sarro. Enfermedad periodontal. } \\
\text { Caries } 27 \text { y } 28 \text {. Hipoplasia del } \\
\text { esmalte } 33 \text { y } 34 \text {. Artrosis en } \\
\text { costillas. Ligera artrosis distal en } \\
\text { cúbito izquierdo. Criba en cuellos } \\
\text { femorales }\end{array}$ & $40-41$ & $52{ }^{\prime} 8$ \\
\hline $\begin{array}{l}\text { Calle de } \\
\text { Herrerías }\end{array}$ & $\begin{array}{l}\text { Musulmán } \\
\text { ss. IX-XI }\end{array}$ & & & & & & \\
\hline $\begin{array}{l}\text { Sepultura } \\
115\end{array}$ & & 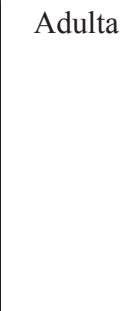 & $\begin{array}{c}\text { M: } \\
\text { 149-150 } \\
\text { T-G: } 150 \\
\end{array}$ & $\begin{array}{c}73 \\
\text { Dolicocráneo }\end{array}$ & $\begin{array}{l}\text { Osteoma parte inferior derecha } \\
\text { del occipital. Sarro. Pérdida } \\
\text { antemortem de } 27 \text {. Caries }(15,16 \text {, } \\
\text { 17, } 24 \text { y } 36) \text {. Osteítis en } 16 \text {. Ligera } \\
\text { impactación de } 38 \text {. Escasa artrosis } \\
\text { en cavidad glenoidea escápula } \\
\text { izquierda. Artrosis costal. Artrosis } \\
\text { T10, T11 y T12. }\end{array}$ & $22-23$ & 34 \\
\hline $\begin{array}{l}\text { Sepultura } \\
185\end{array}$ & & Adulta & $\begin{array}{c}\mathrm{M}: \\
151-152 \\
\mathrm{~T}-\mathrm{G}: 153\end{array}$ & $\begin{array}{l}67 \\
\text { Braquicráneo }\end{array}$ & $\begin{array}{l}\text { Sarro. Enfermedad periodontal. } \\
\text { Caries en 26. Pérdidas antemortem } \\
16 \text { y 25. Osteítis alveolar } \\
\text { mandibular. Ligera artrosis en la } \\
\text { cavidad glenoidea de la escápula } \\
\text { derecha. Calcificación del } \\
\text { ligamento amarillo T9-T11. Ligera } \\
\text { epifisitis en el borde antero- } \\
\text { superior de de L3, L4 y L5. Ligero } \\
\text { reborde osteofítico en la cara } \\
\text { antero-inferior de L1. Nódulo de } \\
\text { Schmorl en T11. Ligera artrosis en } \\
\text { la articulación proximal de ambos } \\
\text { cúbitos. Artrosis incipiente en la } \\
\text { rótula izquierda. }\end{array}$ & \pm 38 & $49^{\prime} 9$ \\
\hline
\end{tabular}

Figura 8: Principales características de las inhumaciones.

que pudieron asociarse a la muerte de la mujer durante la gestación. Sin embargo, no podemos descartar otras posibles enfermedades, no relacionadas con el embarazo, como las infecciones (respiratorias, sistémicas, etc.), o causas de origen accidental (traumatismos, etc.) que pudieran haber causado los fallecimientos.

De la valoración de otros datos antropológicos como el índice craneal y la talla, observamos que no existe uniformidad. Se han identificado dos mujeres dolicocráneas, una braquicránea y otra mesocránea. Las tallas varían entre 145-146 cm la más baja y 154$155 \mathrm{~cm}$ la más alta, según las tablas de Manouvrier y un centímetro mayores si utilizamos las de Trotter y Gleser.

Dentro del capítulo de las patologías el sarro está presente en todas las mujeres, hecho que se ha de relacionar tanto con dietas ricas en hidratos de carbono, como con la ausencia de una higiene bucal adecuada 
(Campillo, 20001, 340; Chimenos, 2003, 158-159). Es también frecuente la presencia de caries (Gomacin 1; Plaza del castillo 119 y 140; Herrerías 115 y 184), y de pérdidas dentales antemortem (Gomacin 1, Herrerías 115 y 184), entre otras patologías estomacales (Fig. 8).

Dentro de la patología tumoral benigna destacamos la presencia de un osteoma en el cóndilo mandibular derecho (Plaza del Castillo 119), y de otro localizado en la parte inferior derecha del occipital (Herrerías 115). Es posible que ninguno de ellos tuviera repercusión sobre el estado de salud de las mujeres.

En general los esqueletos pertenecen a mujeres adultas jóvenes aunque se identifican signos de artrosis tanto en huesos largos como en algunas vértebras. Destacaremos la existencia de nódulos de Schmorl en la sepultura 119 de la Plaza del Castillo y en la 184 de Herrerías. Los signos de artrosis, las calcificaciones del ligamento amarillo y algunas entesopatías identificadas suelen tener relación con la edad, aunque en nuestra muestra creemos que deben relacionarse más con la realización de trabajos físicos de forma reiterada. Este hecho debe ser relacionado con los hábitos de vida esperados para poblaciones de la Alta Edad Media.

\section{CONCLUSIONES}

Aunque no deja de ser anecdótica la presencia de mujeres embarazadas en contextos arqueológicos, creemos relevante que se vayan publicando los casos, cada vez más numerosos, identificados durante las excavaciones.

En este estudio presentamos cinco procedentes de tres necrópolis navarras, una visigoda (Gomacin, Puente la Reina) y dos islámicos (Plaza del Castillo, Pamplona; Calle Herrerías, Tudela).

Todas las mujeres fallecieron a edad adulta. La edad fetal varía documentándose un feto inmaduro de 22-23 semanas (Herrerías 115) y otros cuatro a término (38-41 semanas). Hemos propuesto diferentes causas relacionadas con el embarazo que pudieran haber causado la muerte de las gestantes y consecuentemente la de los fetos (placenta previa, desprendimiento de placenta, gestosis, infecciones, etc.), no descartando posibles distocias pelvico-cefálicas en el caso de las gestaciones a término.

Las patologías presentes en los esqueletos de las mujeres son, en general, las esperadas en poblaciones medievales (caries, pérdidas antemortem, sarro, etc.). Igualmente, la existencia de artrosis y entesopatías relacionadas con actividades físicas forzadas, propias del estilo de vida de poblaciones preindustriales. En ningún caso se han identificado patologías que puedan considerarse causantes de la muerte de ninguna de ellas.

Los índices craneales son variados siendo una braquicránea, dos dolicocráneas y otra mesocránea. No ha sido nuestra intención realizar una clasificación tipológica sino la de ofrecer unos datos que estaban a nuestra disposición.

La altura en los cinco casos estaría dentro de la talla mediana, situándose la de la sepultura 119 al límite de ser considerada como talla baja.

Esperamos poder ampliar este estudio dado que tenemos referencias de la documentación y excavación de otros casos de gestantes en necrópolis, tanto en $\mathrm{Na}$ varra como en otros contextos peninsulares.

M. Paz de Miguel Ibáñez

pdm@ua.es

\section{BIBLIOGRAFÍA}

AufderheIde, A.C. y Rodríguez-Martín, C., 1998: The Cambridge encyclopedia of human paleopatology, Cambridge.

Ayala JuAN, M.M., 1997: «El Cerro de las Viñas y su área de captación, Coy, Lorca», VII Jornadas de Arqueología Regional, 24-25, Murcia.

AZKARATE GARAI-OlaUn, A., 2007. «La muerte en la Edad Media», La tierra te sea leve. Arqueología de la muerte en Navarra, 177-192, Pamplona.

Begiristain, M.A., ETXeberria, F. y Herrasti, L., 2001: «Tres tumbas de la etapa Hispano-visigoda en Gomacin, Puente la Reina (Navarra)», Cuadernos de Arqueología. Universidad de Navarra, 9, 223-277.

BEgIRISTAIN, M.A., 2007: «Necrópolis de Gomacin (Puente la reina)», La tierra te sea leve. Arqueología de la muerte en Navarra, 203-208, Pamplona.

Bienes CALVO, J.J., 2006: «La Necrópolis Islámica de Herrerías», Revista del Centro de Estudios Merindad de Tudela, 14, 41-61.

BIENES CALVO, J.J., 2007a: «Tudela islámica», en PH. SÉNAC (ed.), Villes et Campagnes de Tarraconaise et d'al-Andalus (VIe-XIe siècle): la transition, 199-218, Zaragoza.

BIENES CALVO, J.J., 2007b: «La Necrópolis islámica de Herrerías (Tudela)», La tierra te sea leve. Arqueología de la muerte en Navarra, 253-258, Pamplona.

Brothwell, D.R., 1987: Desenterrando Huesos. La excavación, tratamiento y estudio de los restos del esqueleto humano, México.

BUIKSTRA, J.E.; UBELAKER, D.H., 1994: Standards for data collection from human skeletal remains, Fayetteville, Arkansas.

CALLEN, P.W., 1997: Ecografia en obstetricia y ginecología, Madrid.

CAMPILlO, D., 2001: Introducción a la paleopatología, Barcelona.

CAmpillo, D. y SubIRÁ, E., 2004: Antropología para arqueólogos, Madrid.

Campillo D., Vilaseca A., Casamitjana E. y AyestaRÁN N., 1998: «Esqueleto de una mujer fallecida por distocia, perteneciente al período tardorromano (Mas Rimbau, Tarragona)», Empúries, 51, 251-256.

CAÑAdA Juste, A., 2002: «Pamplona en el siglo VIII. La historia de los musulanes al hilo de las tumbas halladas 
enla Plaza del Castillo», Diario de Navarra, 3 de marzo, 54-55.

CAPASSO, L., KenNEDY, K.A.R. y WILCZAK, C.A., 1998: Atlas of occupational markers of human remains, Teramo.

CARARACH, V., 1982: «Cesárea. Mortalidad materna», en J. GonzÁlez Merlo y J.R. Del Sol, Obstetricia, 693704, Barcelona.

CODERA Y ZAIDÍN, F., 1903: Estudios críticos de historia árabe española, vol. VII, Colección de Estudios Árabes, Zaragoza.

Chimenos KÜSTNER. E., 2003: «Perspectiva odontoestomatológica en paleopatología», en A. ISIDRO y A. MALGOSA. (ed.), Paleopatología. La enfermedad no escrita, 151-162. Barcelona.

De Miguel IBÁÑEZ, M.P., 2007: «La maqbara de la Plaza del Castillo (Pamplona, Navarra): avance del estudio osteoarqueológico», en PH. SÉNAC (ed.), Villes et Campagnes de Tarraconaise et d'al-Andalus ( $V I^{e}-X I^{e}$ siècle): la transition, 183-197, Zaragoza.

De Miguel IbÁÑEZ, M.P., RodríGUEZ García, I., NAVAS, E., Ávila, M.R. y Mancilla, I., 2007: «Embarazada en la necrópolis de la Puerta Elvira (Granada)», en J. BARCA y J. JiMÉNEZ (eds.), Enfermedad, muerte y cultura en las sociedades del pasado. Importancia de la contextualización en los estudios paleopatológicos, vol. 1, 381-385, Cáceres.

Etxeberria, F., Herrasti, L. y Beguiristain, M.A., 2001: «Signos de lepra en un individuo altomedieval de Navarra», en M. MACIAS y J.E. PICAZO (eds.): La enfermedad en los restos humanos arqueológicos. Actualización conceptual y metodológica, Actas del IV Congreso Nacional de Paleopatología, 319-323, Cádiz.

FARO, J.A., GARcíA-BARBERENA, M. y UnZU, M., 2007: «La presencia islámica en Pamplona», en PH. SÉNAC (ed.), Villes et Campagnes de Tarraconaise et d'al-Andalus (VI ${ }^{e}-X I^{e}$ siècle): la transition, 97-138, Zaragoza.

Faro Carballa, J.A., García-Barberena UnZU, M., UnZu Urmeneta, M. y De Miguel IBÁÑEZ, M.P., 2007: «El cementerio islámico de la Plaza del Castillo (Pamplona)», La tierra te sea leve. Arqueología de la muerte en Navarra, 249-252, Pamplona.

FARo CARballa, J.A., UnZU URMENETA, M., ARMENDÁRIZ AZNAR, R.M. y MATEO PÉREZ, M.R., 2007: «Necrópolis de San Esteban (Beriáin)», La tierra te sea leve. Arqueología de la muerte en Navarra, 227-323, Pamplona.

FAZEKAS, I. y KósA, F., 1978: Forensic Fetal Osteology, Budapest.

Ferembach, D.; SchWidetZKY, I. y SlOUTKAL, M., 1979: «Recommandations pour déterminer l'âge et le sexe sur le squelette», Bull. et Mém. de la Soc. d'Anthrop. de Paris, 6, série XIII, 7-45.

IBN IDARI Al-MARRAKUSI, 1999: Historia de al-Andalus, trad. F. Fernández González, Málaga, (NB trad. de 1860).

Gil PitarCh, P., 2000: «Determinación del sexo», en J.D. Villalaín Blanco y F.J. Puchalt Fortea, Identificación antropológica policial y forense, 141-182, Valencia.

Gómez Bellard, F., 2001: «Estudio Antropológico de las cremaciones», en A. GONZÁLEZ PRATS, La necrópolis de cremación de Les Moreres (Crevillente, Alicante, España), 461-469, Alicante.

LÓPEZ FLORES, I. y MAGARIÑO SÁNCHEZ, M.S., 2007: «Dos casos de embarazos a término con evidencias de distocia procedentes de contextos arqueológicos de época medieval y moderna», Paleopatología, AEP, 4, $10 \mathrm{p}$.

López Seguí, E., Torregrosa, P., Quiles, J., De Miguel, M.P. y NAVARRO, C., 2005: «La necrópolis islámica de l'Alfossar (Novelda, Alicante)», Recerques del Museo d'Alcoi, 14, 143-156.

MAFART, B-Y., 1994: «Approche de la mortalité maternelle au Moyen Âge en Provence», Actes des 6e Journées Anthropologiques, Dossiers de Documentation Archéologique $\mathrm{n}^{\mathrm{o}} 17,207-219$. Paris.

Malgosa, A., Alesan, A., SAfont, S., Ballbe, M. y AyALA, M.M., 2004: «A Dystocic Childbirth in the Spanish Bronce Age», Internacional Journal of Osteoarchaeology, 14, 98-103.

MezQuíriz, M.A., 1965: «Necrópolis visigoda de Pamplona», Príncipe de Viana, 26, 107-131.

ORTNER, D.J., 2003: Identification of pathological conditions in human skeletal remains, San Diego, $2^{\text {a }}$ edición.

Ramos Aguirre, M., 2007: «Necrópolis de Saratsua (Muruzábal)», La tierra te sea leve. Arqueología de la muerte en Navarra, 199-202, Pamplona.

RAscón PÉrez, J., CAMBra-Moo, O. y GonzÁlez MarTíN, A., 2007: «A Multidisciplinary Approach Reveals an Extraordinary Double Inhumation in the Osteoarcheological Record», Journal of Taphonomy, vol. 1, 91-101.

ROSEMBSERG, K., 2005: «Évolution de la parturation», en O. Dutour, J. Hublin y B. VANDERMEersch (eds.), Origine et Évolution des Populations Humaines, 315323, Paris.

UnzU Urmeneta, M., 2004: «Arqueología urbana en Pamplona. La plaza del Castillo: resultados. Polémica de conservación», en A. DomíngUEZ ARRANZ (ed.), Jornadas de Arqueología en suelo urbano, 137-159, Huesca. 